\title{
Properties of Soluble Antigen of Trypanosoma evansi
}

\author{
BY B. S. GILL* \\ Wellcome Laboratories of Tropical Medicine \\ 183 Euston Road, London, N.W.1
}

(Received 3 September 1964)

\begin{abstract}
SUMMARY
Plasma of rats infected with Trypanosoma evansi contained soluble antigen detected by the agar diffusion method. The antigen protected mice against homologous challenge but was not agglutinogenic. Whether the two properties were due to one or two antigens, respectively, was not ascertained. Trypanosome homogenate contained three antigens, two of which were exclusive to the trypanosome, whereas the third antigen was the same as the soluble antigen of the infected rat plasma as established by the gel diffusion and indirect haemagglutination tests.
\end{abstract}

\section{INTRODUCTION}

Weitz $(1960 a, b)$ demonstrated a soluble antigen (exo-antigen) in the serum of rats infected with Trypanosoma brucei; it was detectable by gel diffusion technique, protected immunized mice against homologous challenge and was agglutinogenic. Gray (1960) showed soluble trypanosomal antigens in sera of rats infected with $T$. vivax, $T$. gambiense and $T$. brucei which precipitated with anti-T. vivax sera of cattle. Seed (1963) isolated two antigens from serum of rats infected with $T$. rhodesiense, one a precipitin which lacked immunizing property, and the other which protected the immunized mice but was not detectable by the agar diffusion method. During work on immunity with $T$. evansi, the author observed similar properties in plasma of rats infected with this protozoon, which are reported in this paper.

\section{METHODS}

Strain of the parasite. The Madras strain of Trypanosoma evansi was used which has been described previously (Gill, 1964).

Animals. White mice weighing about $15 \mathrm{~g}$. were used for experiments on immunity. Albino rats of 150-200 g. body weight were used for harvesting trypanosomes for antigen.

Infected rat plasma (IRP). Rats at the height of parasitaemia were anaesthetized with chloroform, and bled from the heart with heparin as anticoagulant. Blood from about ten rats was pooled and centrifuged at $850 \mathrm{~g}$ for $10 \mathrm{~min}$. in centrifuge tubes $18 \mathrm{~cm}$. long and $9 \mathrm{~mm}$. diameter at mouth. The supernatant layer of plasma was pipetted off and immediately Seitz-filtered and stored at $-20^{\circ}$.

Trypanosome homogenate $(\boldsymbol{T H})$. The top thick white layer of trypanosomes left above red cells after removing the infected plasma (IRP) was removed with pipette

* Present address: Indian Veterinary Research Institute, Izatnagar, U.P. India. 
and transferred to a $50 \mathrm{ml}$. tube half-filled with cold buffered glucose saline ( $\mathrm{pH} 7 \cdot 2$ ). The lower pinkish layer of trypanosomes and red cells was similarly removed. The sedimented red cells were resuspended in the glucose saline to original volume and centrifuged as before. The supernatant fluid and the top trypanosome layer were pipetted off and added to the corresponding previous collection. The next pinkish layer was removed and diluted in the glucose saline, added to the similar previous collection and the whole spun as before. The top layer of trypanosomes was collected. The pooled suspension of trypanosomes was counted by the standard haemocytometer technique, and washed thrice in cold distilled water. Trypanosomes were disrupted by two treatments of 15 sec. each with a Mullard type MT 20, MSE ultrasonic disintegrator ( $45 / 60$ cyc./sec., single phase). This constituted the disintegrated trypanosome homogenate which was lyophilized and stored at $-20^{\circ}$. For serological work, $2 \%$ disintegrated trypanosome homogenate in cold normal saline was prepared, as described by Seed (1963).

Anti-IRP serum. Anti-IRP sera were prepared in rats and rabbits, following Weitz $(1960 b)$. Half of the serum samples were frozen immediately at $-20^{\circ}$; the rest was lyophilized and stored at $-20^{\circ}$ and reconstituted with distilled water to the original volume when required.

Anti-trypanosome homogenate sera (anti-TH sera). Rabbits were immunized following the technique described by Seed (1963): two animals each with whole trypanosomes and with trypanosome homogenate, respectively. Rats were immunized by Weitz's $(1960 b)$ method. Sera were absorbed with normal rat plasma proteins as described by Seed (1963).

Gel diffusion test. The Ouchterlony double diffusion method $(1949,1953)$ was used with $1.2 \%$ agar in normal saline containing $1 / 1000$ thiomersalate. The wells were lined with a thin film of agar (Mansi, 1957) and were about $2 \mathrm{~mm}$. deep. The plates were kept in a moist chamber at room temperature (about 17-20 ${ }^{\circ}$.

Agglutination test. The technique, described by Cunningham \& Vickerman (1962), using a glass plate was used.

Haemagglutination test. The indirect haemagglutination test described elsewhere (Gill, 1964) was used to detect antibody in anti-IRP serum of rats. The test antigen used was sonically prepared trypanosome homogenate clarified by centrifugation for $15 \mathrm{~min}$. at $2520 \mathrm{~g}$.

Protection test. To obtain an enhanced immune response, saponin SPL (Johnson, Neal \& Gall, 1963) was used with IRP. Two doses of $0.2 \mathrm{ml}$. plasma mixed with $0 \cdot 1 \mathrm{ml}$. saponin solution $(1 \mathrm{mg} . / \mathrm{ml}$.), a fortnight apart, were given under the neck fold of skin of the mouse. They were challenged intraperitoneally with $1 \times 10^{4}$ trypanosomes/mouse, contained in $\mathbf{0 . 1} \mathrm{ml}$. of heparinized infected mouse blood diluted with normal saline. The challenge dose was given 2 weeks after the last immunizing dose. Tail blood was then daily examined for trypanosomes. Each group had ten mice when challenged. It was observed that the protective effect of the inoculum was reflected in the prepatent and survival periods of each group. Once the infection became patent it ran its course unaffected, resulting in about the same patent period in all groups. Therefore, in the section on Results, patent periods have been omitted. Only the prepatent period of the infection and the longevity after challenge of each group is described. 


\section{RESULTS}

Gel diffusion tests. In agar gel two precipitation bands, one prominent and the other faint, appeared between IRP and anti-IRP serum of rats and rabbits. The anti-IRP sera, however, did not precipitate with normal rat plasma. These observations indicated the presence of a soluble antigen in the IRP. To prove the specificity of this antigen and to elucidate its relationship with antigens of the whole trypanosome, anti-IRP sera and anti-TH serum were side by side titrated against IRP. Results of this experiment are shown in Fig. 1, which has been constructed from the agar plate photographed at intervals to show all the precipitation bands appearing at different speeds.

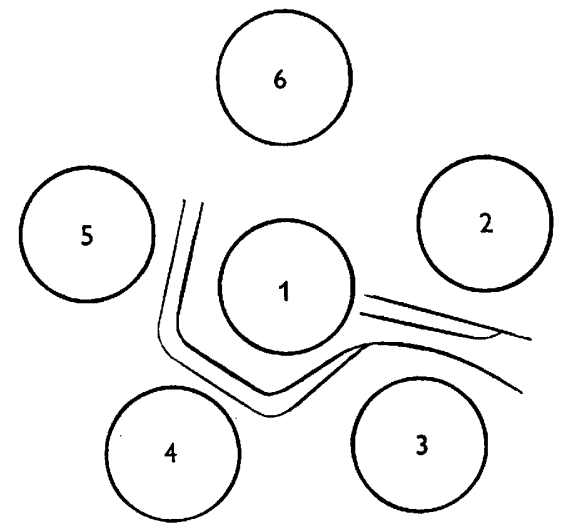

Fig. 1. Double gel diffusion precipitin reactions of the soluble antigen and homogenate of Trypanosoma evansi with their antisera. 1. Plasma of rats infected with T. evansi. 2. Trypanosome homogenate ( $T$. evansi). 3. Anti-trypanosome homogenate ( $T$. evansi) serum prepared from rabbit and absorbed with lyophilized normal rat plasma. 4. AntiIRP serum prepared from rats. 5. Anti-IRP serum prepared from rabbit and absorbed with lyophilized normal rat plasma. 6. Anti-IRP serum of rats absorbed with lyophilized trypanosomes ( $T$. evansi).

Figure 1 shows that three bands were formed by the reaction of trypanosome homogenate and anti-TH serum of rabbit absorbed with normal rat plasma proteins, indicating the presence of at least three antigens in the trypanosome. Two bands were precipitated between IRP and anti-TH serum of rabbit, which were identical with the two bands between the IRP and anti-IRP sera. This reveals the presence of the antibody to the soluble antigen both in anti-IRP and anti-TH sera. The two bands due to the soluble antigen at the other end joined one of the three bands of the TH-anti-TH reaction, thus establishing the identity of the soluble antigen with one of the three antigens in the trypanosome homogenate. It appears that this band between TH and anti-TH serum represented two antigen-antibody factors which were resolved into two identical bands between IRP and anti-IRP serum owing perhaps to better conditions present between the latter reactant wells. That the bands represented true trypanosomal antigen-antibody reactions is conclusively proved since (1) the antisera did not contain antibodies to the normal rat plasma as shown by the lack of precipitation line against the normal rat proteins; (2) the absorption of antisera with lyophilized trypanosomes neutralized the anti- 
body completely as absorbed sera failed to precipitate with both IRP and try. panosome homogenate. These observations established the identity of the soluble antigen in the plasma of rats infected with Trypanosoma evansi with one of the three antigens present in the whole trypanosome.

Agglutination test. Anti-TH sera of two rabbits gave an agglutinating titre of 1/80. The anti-IRP sera, however, did not agglutinate trypanosomes.

Table 1. Prepatent period and survival time of mice immunized with normal rat plasma and infected rat plasma with or without saponin SPL as adjuvant

Mice were challenged with $1 \times 10^{4}$ trypanosomes a fortnight after last immunizing dose. Each group consisted of ten mice when challenged with trypanosomes.

\begin{tabular}{clcc} 
Group & \multicolumn{1}{|c}{$\begin{array}{c}\text { Average } \\
\text { prepatent } \\
\text { period } \\
\text { (days) }\end{array}$} & $\begin{array}{c}\text { Average } \\
\text { survival time } \\
\text { after challenge } \\
\text { (days) }\end{array}$ \\
1 & No treatment & $1 \cdot 5$ & $5 \cdot 0$ \\
2 & Saponin SPL alone & $1 \cdot 4$ & $5 \cdot 0$ \\
3 & Infected rat plasma (IRP) alone & $\mathbf{5 \cdot 4}$ & $\mathbf{8 \cdot 9}$ \\
4 & IRP + saponin SPL & $7 \cdot 2$ & $10 \cdot 8$ \\
5 & Normal rat plasma (NRP) & $1 \cdot 4$ & $4 \cdot 9$ \\
6 & NRP + saponin SPL & $1 \cdot 4$ & $5 \cdot 1$
\end{tabular}

Haemagglutination test. The anti-IRP serum from rats gave a titre of $1 / 9000$ against the entire soluble antigen complex contained in the trypanosome homogenate. This observation lends further support to the conclusion derived from gel diffusion test that the soluble antigen of the plasma of rats infected with Trypanosoma evansi formed a part of the antigenic complex of the trypanosome.

Protection test. Results of the investigation on the protective nature of immunity conferred by the soluble antigen of the IRP are summarized in Table 1. The data given in Table 1 show that the prepatent period of the infection in mice immunized with IRP was significantly longer than that of the unprotected control mice (cf. the prepatent period of $5 \cdot 4$, and 1.4 to 1.5 days, respectively). Therefore, it was concluded that the soluble antigen in the plasma of rats infected with Trypanosoma evansi excited the development of immunity which was of a protective character. The immunogenicity of the IRP was considerably enhanced when injected with the adjuvant, saponin SPL, as the prepatent period of the infection in mice immunized thus was further increased to $\mathbf{7 \cdot 2}$ days.

\section{DISCUSSION}

The whole operation of collection of infected blood from rats and separation of plasma from trypanosomes and its filtration was achieved as rapidly as possible $(20 \mathrm{~min}$.) to minimize the possibility of 'contamination' of the plasma with products of disintegrating trypanosomes which might have died after leaving the host. During this time, appreciable number of trypanosomes could not have died since microscopic examination of plasma before filtration showed only active forms. It is therefore reasonable to assume that whatever antigenic trypanosomal factor or 
factors were detected in the infected plasma were already there in the blood before it was taken from the rats. It appears that the soluble antigen of the infected plasma might have been secreted or excreted by the living trypanosomes, since this antigen also formed a part of the whole trypanosome antigen as detected by the gel diffusion and haemagglutination tests. The soluble antigen of Trypanosoma evansi was precipitinogenic, and partially protected mice against infection with the trypanosome. Whether a single soluble antigen in plasma of rats infected with $T$. evansi excited the development of precipitin and protective antibody, or whether two distinct antigens were involved, as in $T$. rhodesiense (Seed, 1963), was not determined. The antigen was not agglutinogenic.

The author wishes to thank Dr R. A. Neal, Head of Protozoology Department, Wellcome Laboratories of Tropical Medicine (in whose laboratory the work was done) for his interest in the work, and the Nuffield Foundation, London, for the award of a Dominion Travelling Fellowship in Natural Sciences.

\section{REFERENCES}

Cunningham, M. P. \& Vickerman, K. (1962). Antigenic analysis in the Trypanosoma brucei group, using the agglutination reaction. Trans. Roy. Soc. trop. Med. Hyg. 56, 48.

GiLL, B. S. (1964). Indirect haemagglutination technique in the study of experimental Trypanosoma evansi infections. Ann. trop. Med. Parasit. 58, (in the Press).

GraY, A. R. (1960). Soluble antigens of Trypanosoma vivax and of other trypanosomes. Immunology, 4, 253.

Johnson, P., Neal, R. A. \& Gal, D. (1963). Protective effect of killed trypanosome vaccines with incorporated adjuvants. Nature, Lond. 200, 83.

MansI, W. (1957). The study of some viruses by the plate gel diffusion precipitin test. J. comp. Path. 67, 297.

Ouchterlony, O. (1949). Antigen-antibody reactions in gels. Ark. Kemi Miner. Geol. 26 B, 1. (Article 14.)

Ouchterlony, O. (1953). Antigen-antibody reactions in gels. IV. Types of reactions in coordinated systems of diffusion. Acta path. microbiol. scand. 32, 231.

SEED, R. (1963). The characterisation of antigens isolated from Trypanosoma rhodesiense. J. Protozool. 10, 380.

Wertz, B. (1960a). A soluble protective antigen of Trypanosoma brucei. Nature, Lond. 185, 788.

Wertz, B. (1960b). The properties of some antigens of Trypanosoma brucei. J. gen. Microbiol. 23, 589. 
